\title{
Regional differences of vitamin D deficiency in rheumatoid arthritis patients in Italy
}

\author{
M. Rossini' ${ }^{1}$, G. D'Avola ${ }^{2}$, M. Muratore ${ }^{3}$, N. Malavolta ${ }^{4}$, F. Silveri', G. Bianchi', \\ B. Frediani ${ }^{7}$, G. Minisola ${ }^{8}$, M.L. Sorgi ${ }^{9}$, M. Varenna ${ }^{10}$, R. Foti ${ }^{11}$, G. Tartarelli ${ }^{12}$, \\ G. Orsolini' ${ }^{1}$ S. Adami ${ }^{1}$ on behalf of Study Group on Osteoporosis \\ and Metabolic Skeletal Diseases of the Italian Society of Rheumatology (SIR) \\ ${ }^{1}$ Rheumatology Unit, University of Verona; ${ }^{2}$ Rheumatology Unit, AUSL 3, Catania; \\ ${ }^{3}$ Rheumatology Unit, Galateo San Cesario Hospital, Lecce; ${ }^{4}$ Rheumatology Unit, University of Bologna; \\ ${ }^{5}$ Rheumatology Unit, University of Ancona, Jesi (AN); ${ }^{6}$ Rheumatology Unit, La Colletta Hospital, \\ Arenzano (GE); ${ }^{7}$ Rheumatology Unit, University of Siena; ${ }^{8}$ Rheumatology Unit, San Camillo Hospital, Roma; \\ ${ }^{9}$ Rheumatology Unit, University "La Sapienza", Roma; \\ ${ }^{10}$ Rheumatology Unit, Gaetano Pini Orthopedic Institute, Milano; \\ ${ }^{11}$ Rheumatology Unit, Vittorio Emanuele Hospital, Catania; \\ ${ }^{12}$ Rheumatology Unit, Hospital of Massa (MS), Italy
}

\section{SUMMARY}

Vitamin D deficiency is very common in patients with rheumatoid arthritis (RA). Aim of this study was to evaluate the prevalence of vitamin D deficiency among the different Italian regions and whether these variations are associated with different severity of the disease.

The study includes 581 consecutive RA patients (464 women), not taking vitamin D supplements, from 22 Italian rheumatology centres uniformly distributed across Italy. Together with parameters of disease activity (disease activity score 28), functional impairment (activities of daily living and health assessment questionnaire disability index) and mean sun exposure time, all patients had serum 25-hydroxyvitamin D (25OHD) measured in a centralized laboratory.

Vitamin D deficiency (25OHD level $<20 \mathrm{ng} / \mathrm{mL}$ ) was very frequent among RA patients; its prevalence was $60 \%, 52 \%$ and $38 \%$ in southern, central and northern Italy, respectively. Mean disease activity and disability scores were worse in southern regions of Italy. These scores were inversely related to 25OHD levels and this correlation remained statistically significant after adjusting for both body mass index (BMI) and sun exposure time. However, disease severity remained significantly higher in southern regions versus central-northern Italy after adjustment also for serum $250 H D$ levels, age and BMI.

In RA Italian patients there are significant regional differences in the prevalence of vitamin D deficiency explained by different BMI, and sun exposure time, and inversely associated with disease activity and disability scores.

Key words: Rheumatoid arthritis, Vitamin D, Disease activity, Disability, Body mass index.

Reumatismo, 2013; 65 (3): 113-120

\section{INTRODUCTION}

$\mathrm{V}$ itamin D deficiency is extremely common in Europe and particularly in Southern countries $(1,2)$, not only in elder people but also among young subjects (3). Recently in an Italian multicentric study (4), involving more than one thousand patients affected by rheumatoid arthritis (RA), we showed that more than half of the patients was vitamin D deficient, defined as serum 25-hydroxyvitamin D (25OHD) concentration $<20 \mathrm{ng} / \mathrm{mL}(5$,
6). In the same study (4) we confirmed that the main determinants of serum vitamin D level are also in RA patients body mass index (BMI) and sun exposure time. A significant inverse correlation was found between 25OHD serum level and disease activity or disability scores (4), while bone erosions were associated with higher parathyroid hormone levels (7). Similar findings in smaller cohort of patients were reported by other authors (8-10), but not all (11-13). The causal link between vitamin D deficiency and disease severity could not
Corresponding author: Maurizio Rossini Rheumatology Unit, University of Verona Policlinico Borgo Roma

Piazzale Scuro, 10 - 37134 Verona, Italy E-mail: maurizio.rossini@univr.it 
be ascertained since sun exposure time was also lower in these patients and sun exposure may be dependent on disease severity and then the propensity to stay outside.

The aim of this sub-analysis of the Italian multicentric study (4) is to evaluate the regional differences in the prevalence of vitamin D deficiency, and whether these are associated with differences in the activity of the disease.

\section{MATERIALS AND METHODS}

\section{Patients}

This is a subgroup analysis of the patients not taking vitamin D supplements and participating in the Italian multicentre study on vitamin D status in RA patients, sponsored by the Italian Rheumatology Society (4). The study population includes 581 consecutives patients (464 females, 117 males) aged between 30 and 75 years afferent to 22 rheumatologic clinics equally dis-

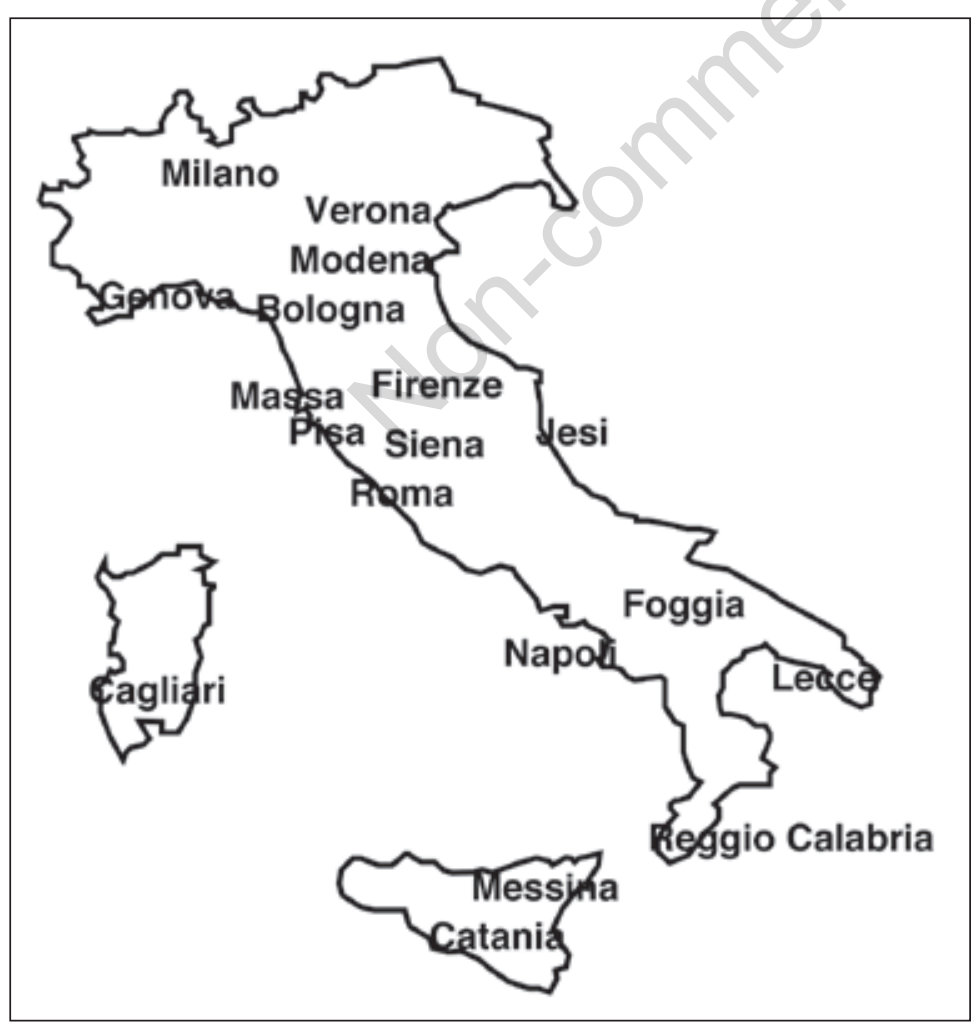

Figure 1 - Geographic distribution of the centres participating in the study. tributed over Italy ( 6 in the North, 8 in the Center and 8 in the South; Figure 1). The diagnosis of RA was made according to the American College of Rheumatology 1987 criteria (14). Subjects with comorbilities such as insulin-dependent diabetes or severe renal or hepatic failure were excluded. The study was in compliance with the Helsinki Declaration and was approved by the local Ethical Committees. An informed written consent was obtained from all participants.

\section{Clinical evaluation}

All patients were interviewed and examined at each clinical center for the gathering of information on disease and treatment history. Disease-related variables included disease onset and duration, presence of extra-articular manifestations, 28 tender joint count (TJC28) and 28 swollen joint count (SJC28). The three-variable disease activity score (DAS 28) was calculated using C-reactive protein (CRP) and the Nijmegen formula (15): DAS2 $8=(0.56 * \operatorname{sqrt}(\mathrm{TJC} 28)$ $+0.28 *$ sqrt $(\mathrm{SJC} 28)+0.36 * \ln (\mathrm{CRP}+1))$ $* 1.10+1.15$. Clinical measures of disease related functional impairment included health assessment questionnaire disability index (HAQ), and the mobility activities of daily living (ADL). RA specific treatment details were collected for glucocorticoids, disease modifying anti-rheumatic drugs: methotrexate, cyclosporine, gold salts, sulfasalazine, antimalarials, and azathioprine, and the tumor necrosis factor- $\alpha$ blockers (anti-TNF). Patients were interviewed regarding current use of drugs affecting bone metabolism including bisphosphonates, calcium and vitamin D supplements.

Exposure to sunlight from March to September (sun exposure time) was quantified with a semiquantitative scale from 0 to 3 as follow: $<10,10$ to 20,20 to 30 or $>30$ min daily (4). Body weight and height (Harpender stadiometer) were assessed and the BMI $\left(\mathrm{kg} / \mathrm{m}^{2}\right)$ was calculated in all subjects.

\section{Laboratory assessment}

Rheumatoid factor, anti-cyclic citrullinated peptide and routine biochemistry were 


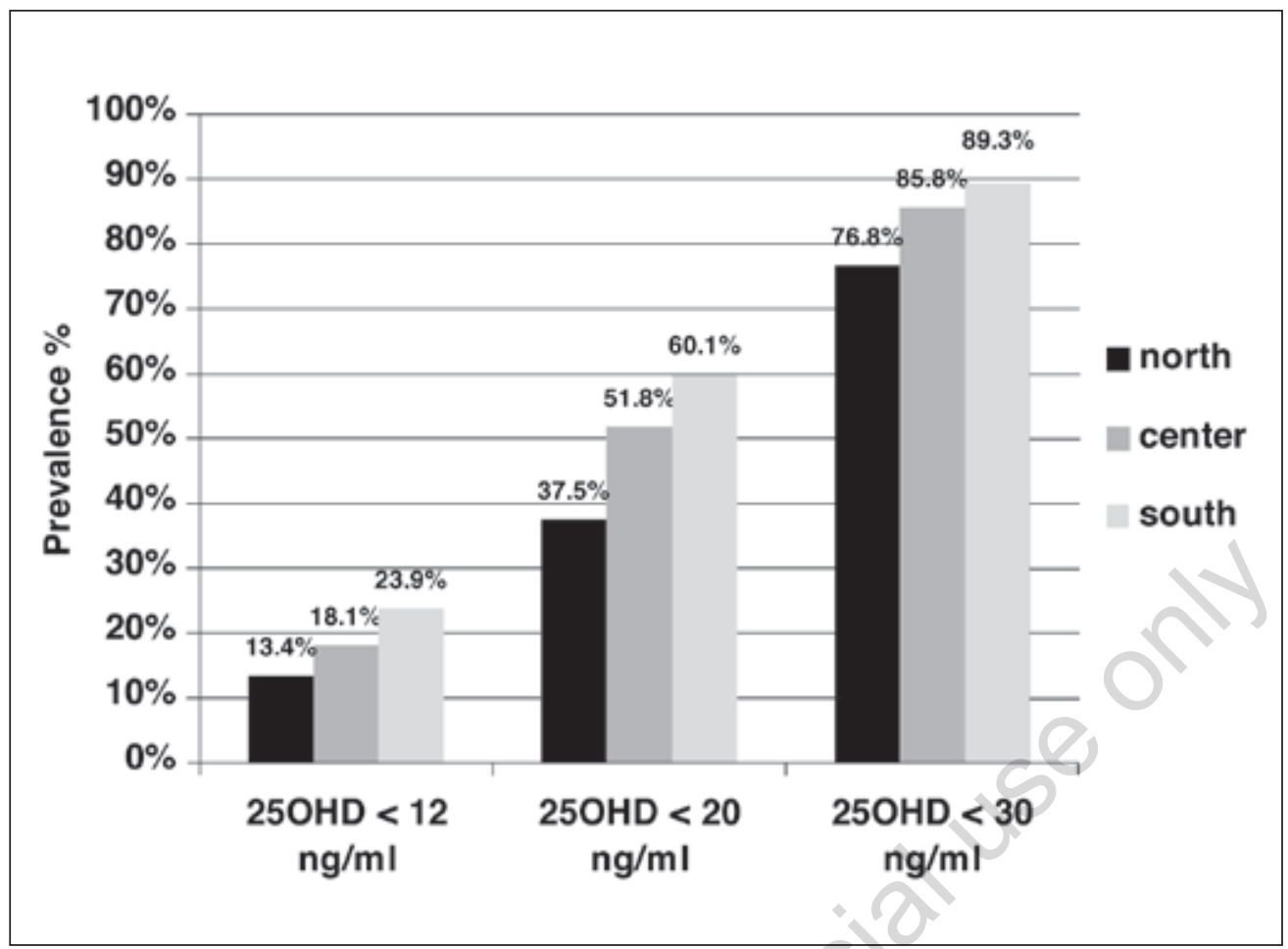

Figure 2 - Prevalence of hypo-vitaminosis D in the north, center and south of Italy. 25OHD, 25-hydroxyvitamin D.

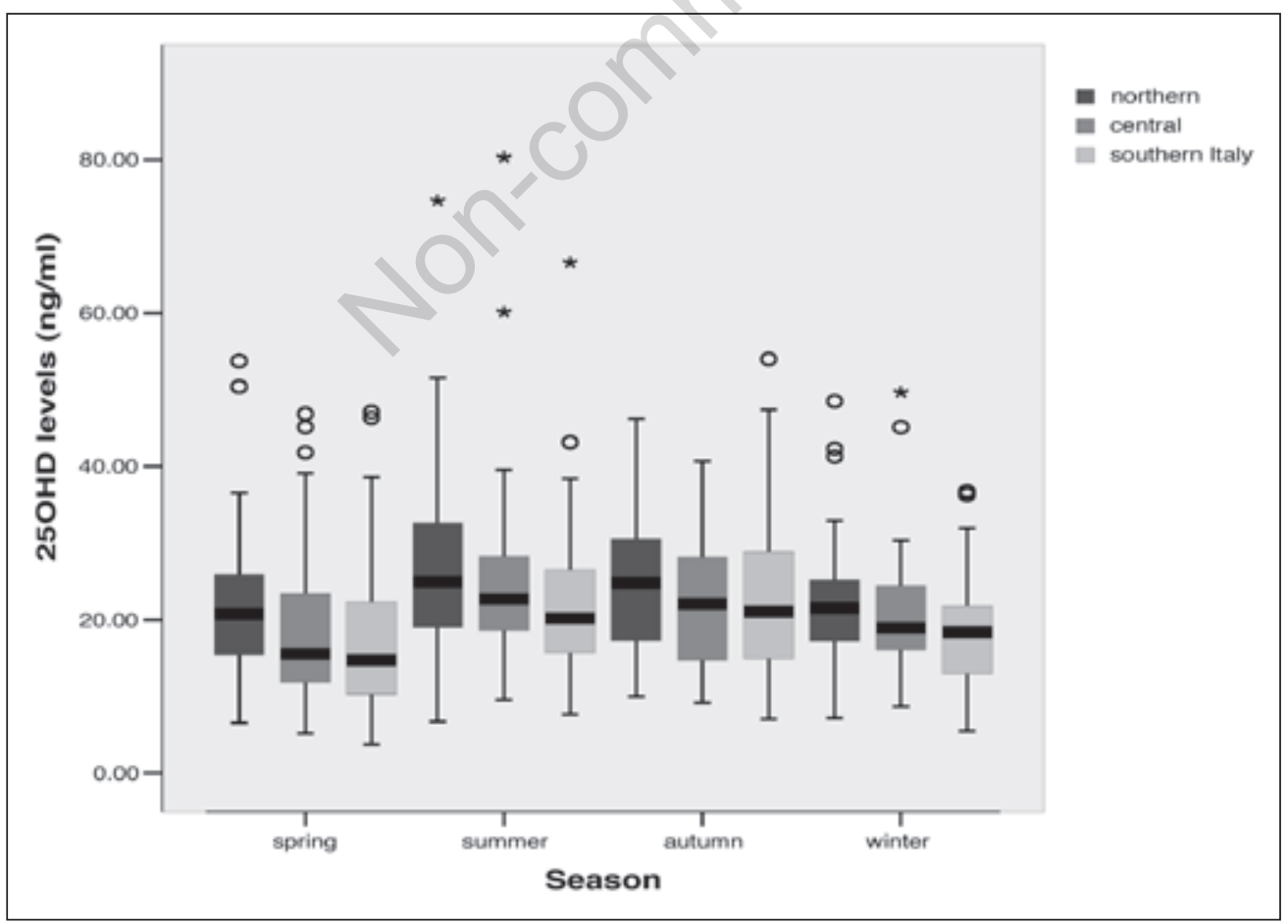

Figure 3 - Seasonal variations of 25-hydroxyvitamin D (25OHD) serum level (box-plots) in the north, center and south of Italy. 
measured locally. Serum samples were collected from June 2007 to May 2008 from each patient and 4 aliquots were sent on dry ice by courier to the laboratory of the University of Verona, and kept at $-70^{\circ} \mathrm{C}$ until the measurement of serum 25OHD using commercial ELISA kits (IDS Co., Bolden, UK) with inter-assay coefficient of variations ranging from 5 to $15 \%$.

\section{Statistical analysis}

All data management and analysis were centralized and conducted by one of the centres. The differences between subgroups were assessed by t-test or analysis of variance (ANOVA); analysis of covariance (ANCOVA) was used to adjust values for confounding factor. Chi-square tests were used for categorical data.

Associations between continuous variables were examined using Pearson correlation coefficients ( $r$ ) and multivariate linear regression. Differences were considered significant at $\mathrm{P}<0.05$.
All statistical procedures were carried out using a medical statistics computer program (SPSS version 13.0, Inc., Chicago, IL, USA).

\section{RESULTS}

The study patients were divided in three subgroups by regions of residence in the north, the center or the south of Italy. The mean age ( $56 \pm 11$ years) and disease duration (132 \pm 105 months) were not significant different in the 3 subgroups of patients. Similar were also the seasonal distribution of serum sampling and the RA specific treatment.

Significant differences between north-center and south were observed in BMI (25.0 vs 25.8 , respectively, $\mathrm{P}=0.033$ ) and in degree of sun exposure time score (1.8 vs 1.6, respectively, $\mathrm{P}=0.02$ ).

The prevalence of vitamin $\mathrm{D}$ deficiency $(25 \mathrm{OHD}<20 \mathrm{ng} / \mathrm{mL})$ was more common

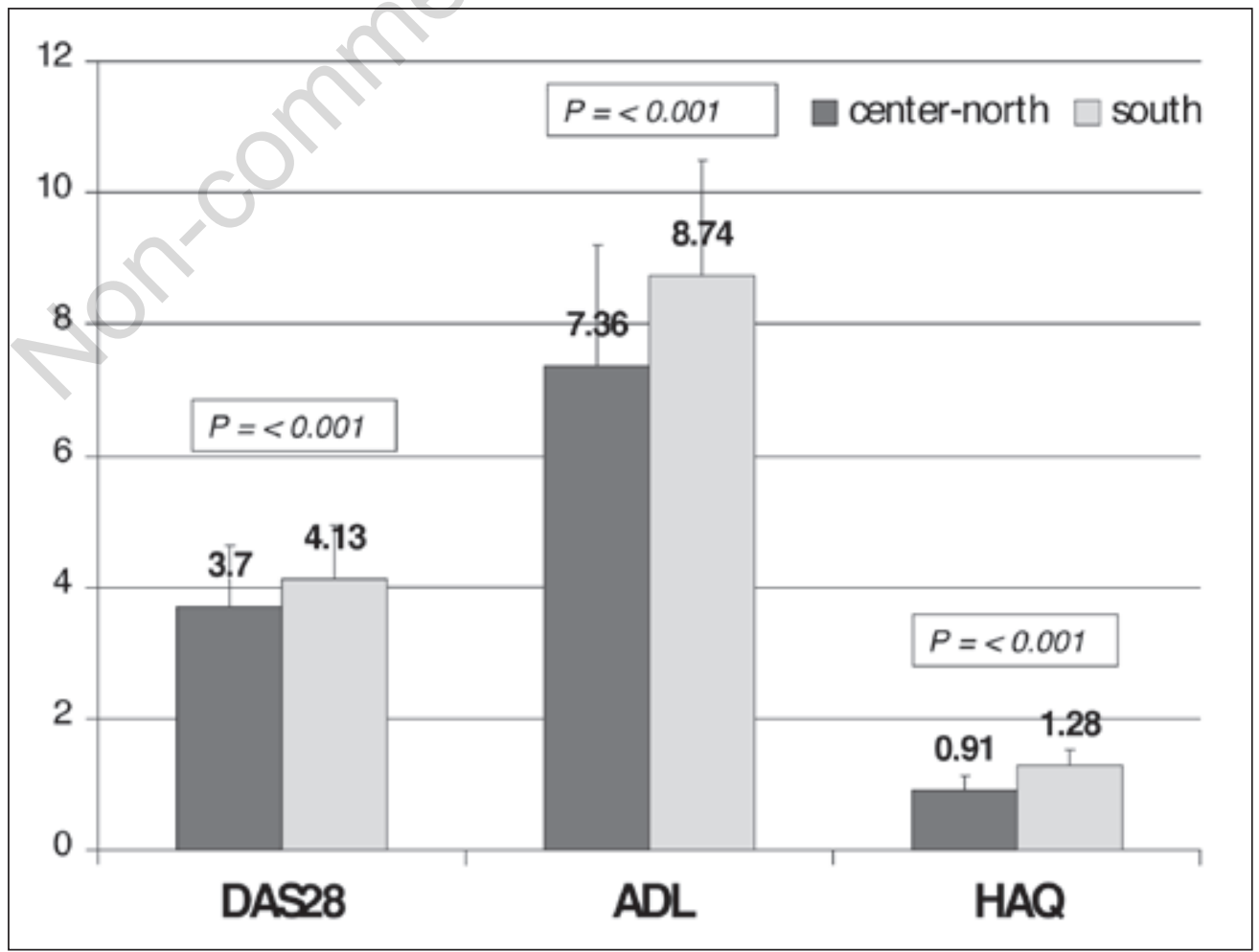

Figure 4 - Disease activity score 28 (DAS28), activities of daily living (ADL) and health assessment questionnaire disability index $(\mathrm{HAQ})\left(\mathrm{mean}_{\mathrm{S}} \mathrm{SE}\right)$ in the north-center and south of Italy. 
$(\mathrm{P}<0.01)$ in the south and centre than in the north $(60.1 \%, 51.8 \%$, and $37.5 \%$, respectively) (Fig. 2).

The mean 25OHD levels in each macroregion are shown in Figure 3, according with the season of blood collection: a seasonal trend was apparent but the lower mean 25OHD serum levels in the south were confirmed $(\mathrm{P}<0.05)$ also for values adjusted for the month of blood collection. The mean DAS 28, HAQ and ADL scores were higher in the south as compared to center-north regions (4.1 vs $3.7 ; 1.28$ vs $0.91 ; 8.74$ vs 7.36 , respectively; $\mathrm{P}<0.001$ ) (Fig. 4).

25OHD serum level correlates negatively with age $(\mathrm{P}<0.05)$ and BMI $(\mathrm{P}<0.001)$, and positively with sun exposure time $(\mathrm{P}<0.001)$.

Negative significant correlations were found between 25OHD levels and HAQ $(\mathrm{r}=-0.20 ; \mathrm{P}<0.001)$ or DAS28 $(\mathrm{r}=-0.17$, $\mathrm{P}<0.001$ ) (Fig. 5). These correlations remained significant after correction for the determinants of vitamin D status, such as $\mathrm{BMI}$ and sun exposure time.

The mean DAS 28, HAQ and ADL scores remained significantly higher in the south as compared to northern and central regions $(\mathrm{P}<0.001)$ also after adjustment for 25OHD serum levels, age and BMI.

\section{DISCUSSION}

It was reported that the risk of vitamin D deficiency rises with the latitude possibly as a consequence of lower number of hours of efficient sunlight (16). In this study we observed that RA patients living in southern Italy are at higher risk of hypo-vitaminosis D than patients living in central-northern Italy, despite the average latitude was 38 and 45 for the rheumathology centres of southern and northern regions, respectively. Seasonal variation of $25 \mathrm{OHD}$ serum level was somewhat more visible in the south than in the north, with the expected nadir values at the end of winter and at the beginning of spring.

Interestingly, similar observations were reported in 4 surveys on elderly populations of Europe, with the prevalence of vitamin $\mathrm{D}$ insufficiency being higher in southern Europe (1, 2, 17, 18).

Our findings may be explained at least in part by the significantly higher mean BMI values observed in patients from south Italy, since BMI is known to be inversely associated to serun levels of 25OHD (4). Another explanation is that more hours of sunlight do not mean necessarily longer sun exposure! During summer but also spring, sunlight is so burning that traditionally people

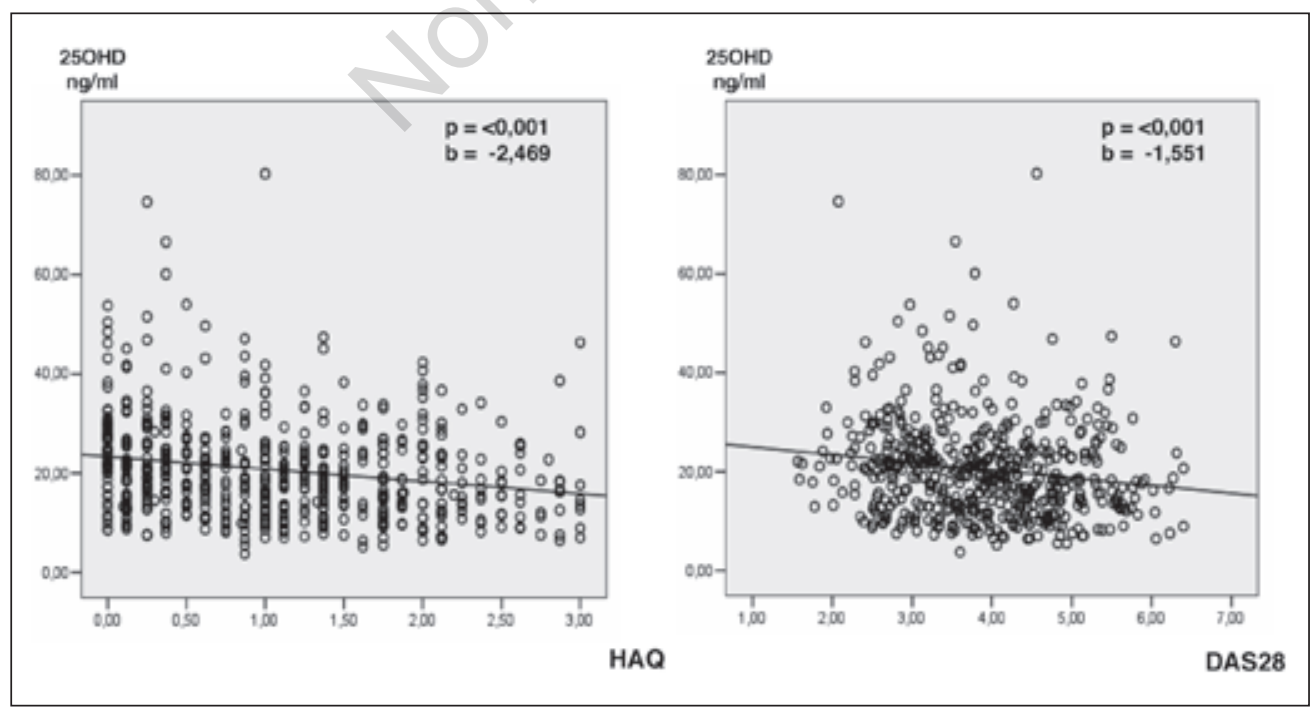

Figure 5 - Correlations between 25-hydroxyvitamin D (25OHD) serum levels, health assessment questionnaire disability index (HAQ) and disease activity score 28 (DAS28). 
living in southern Mediterranean regions avoid sunlight exposure. Indeed, in our patients series sun exposure was significantly shorter in the south as compared to centernorth Italy.

In our study we observed that both disease activity and disability scores were worst in patients living in the southern regions. Of interest are the observed correlations between 25OHD serum levels and disease activity (DAS28) and disability scores (HAQ and ADL) that remained significant after correction for BMI (data not shown).

The explanation of this finding is not clear since we are not in the condition to control for a number of factors such as the selection of the patients included in the study and the average standard of care. However these correlations remained significant also by analyzing only southern patients. Twelve studies evaluated the association between 25OHD levels and RA (4, 8-13, 19-23).

Nine of these studies showed that 250HD serum levels are inversely associated with RA activity, such as, DAS28. It is conceivable that patients with more severe disease are also likely to spend less hours out-door, and that this may lead to lower 25OHD levels in not supplemented patients, but the 25OHD difference between south and north Italy and the correlation between 25OHD levels and disease activity or functional impairment, remained statistically significant also when the 25OHD levels were adjusted for sun exposure time.

This latter observation might be explained by the recent report that $25 \mathrm{OHD}$ serum concentration decreases in inflammatory conditions (24) generating a vicious circle where inflammation is responsible of lower 25OHD levels which, in turn, might lead to worsening of the disease as suggested by the multiple effects of vitamin D on immune system (25) and muscle performance (26).

Our findings are far from conclusive in establishing a causal relationship between low 25OHD levels and disease severity in RA patients. We have been unable to control the clinical data for the standard of care and we cannot exclude that patients seen in the secondary care centers of the south of Italy are more selected for their severity. The causality link between low 25OHD levels and disease severity can be determined only with longitudinal intervention studies.

Nevertheless the mean DAS 28, HAQ and ADL scores remained significantly higher in the south as compared to northern and central regions also after adjustment for 25OHD serum levels. The poorer control of disease activity in southern Italy is likely to be related to other factors.

Recently, a deleterious effects of higher BMI on clinical control of RA (27) and on the response to treatment (28) was reported and, indeed, in our study significantly higher BMI values were observed in southern regions, but in a multivariate analysis the correlation of BMI with clinical scores was not significant.

In conclusion in RA Italian patients there are significant regional differences in the prevalence of vitamin D deficiency and these are associated with different disease activity and disability scores.

Even though a causal link between vitamin $\mathrm{D}$ deficiency and RA severity remains to be established, our results indicate that vitamin D supplementation must be recommended to all patients even if only for the prevention of secondary osteoporosis (29).

Acknowledgments: the authors would like to thank the centers participating in this study (in alphabetical order): Silvano Adami (Verona), Gianfilippo Bagnato (Messina), Gerolamo Bianchi (Genova), Enrico Cacace (Cagliari), Maurizio Caminiti (Reggio Calabria), Francesco Cantatore (Foggia), Giovanni D'Avola (Catania), Antonio Del Puente (Napoli), Ombretta Di Munno (Pisa), Clodoveo Ferri (Modena), Rosario Foti (Catania), Bruno Frediani (Siena), Anna Maria Iagnocco (Roma), Giovanni La Montagna (Napoli), Susanna Maddali Bongi (Firenze), Nazzarena Malavolta (Bologna), Giovanni Minisola (Roma), Maurizio Muratore (Lecce), Ferdinando Silveri (Ancona), Luigi Sinigaglia (Milano), Maria Laura Sorgi (Roma), Giancarlo Tartarelli (Massa). 
Conflict of interests: the authors declare no conflict of interests.

Funding: this study was partially supported by an unlimited and unrestricted grant from Merck Sharp \& Dohme, Italia. A further support was also obtained by the Italian Society of Rheumatology (SIR).

\section{REFERENCES}

1. Kuchuk NO, van Schoor NM, Pluijm SM, et al. Vitamin D status, parathyroid function, bone turnover, and BMD in postmenopausal women with osteoporosis: global perspective. J Bone Miner Res. 2009; 24: 693-701.

2. Wahl DA, Cooper C, Ebeling PR, et al. A global representation of vitamin D status in healthy populations. Arch Osteoporos. 2012; 7: $155-72$.

3. Adami S, Bertoldo F, Braga V, et al. 25-hydroxy vitamin $\mathrm{D}$ levels in healthy premenopausal women: association with bone turnover markers and bone mineral density. Bone. 2009; 45: 423-6.

4. Rossini M, Maddali Bongi S, La Montagna $\mathrm{G}$, et al. Vitamin D deficiency in rheumatoid arthritis: prevalence, determinants and associations with disease activity and disability. Arthritis Res Ther. 2010; 12: R216.

5. Holick MF, Binkley NC, Bischoff-Ferrari HA, et al. Evaluation, treatment, and prevention of vitamin D deficiency: an endocrine society clinical practice guideline. J Clin Endocrinol Metab. 2011; 96: 1911-30.

6. Adami S, Romagnoli E, Carnevale V, et al. Guidelines on prevention and treatment of vitamin D deficiency. Reumatismo. 2011; 63: 129-47.

7. Rossini M, Bagnato G, Frediani B, et al. Relationship of focal erosions, bone mineral density, and parathyroid hormone in rheumatoid arthritis. J Rheumatol. 2011; 38: 997-1002.

8. Cutolo M, Otsa K, Laas K, et al. Circannual vitamin $\mathrm{d}$ serum levels and disease activity in rheumatoid arthritis: Northern versus Southern Europe. Clin Exp Rheumatol. 2006; 24: 702-4.

9. Patel S, Farragher T, Berry J, et al. Association between serum vitamin $\mathrm{D}$ metabolite levels and disease activity in patients with early inflammatory polyarthritis. Arthritis Rheum. 2007; 56: 2143-49.

10. Turhanog lu AD, Güler H, Yönden Z, et al. The relationship between vitamin $\mathrm{D}$ and disease activity and functional health status in rheumatoid arthritis. Rheumatol Int. 2011; 31: 911-4.

11. Oelzner P, Muller A, Deschner F, et al. Rela- tionship between disease activity and serum levels of vitamin D metabolites and PTH in rheumatoid arthritis. Calcif Tissue Int. 1998; 62: 193-8.

12. Craig SM, Yu F, Curtis JR, et al. Vitamin D status and its associations with disease activity and severity in African Americans with recentonset rheumatoid arthritis. J Rheumatol. 2010; 37: 275-81.

13. Baker JF, Baker DG, Toedter G, et al. Associations between vitamin $\mathrm{D}$, disease activity, and clinical response to therapy in rheumatoid arthritis. Clin Exp Rheumatol. 2012; 30: 658-64.

14. Arnett FC, Edworthy SM, Bloch DA, et al. The American Rheumatism Association 1987 revised criteria for the classification of rheumatoid arthritis. Arthritis Rheum. 1988; 31: 315-24.

15. Das-Score NL. Available from: http://www. das-score.nl/das28/en/

16. Adams JS, Clemens TL, Parrish JA, Holick MF. Vitamin-D synthesis and metabolism after ultraviolet irradiation of normal and vitamin-D-deficient subjects. N Engl J Med. 1982; 306: 722-5.

17. van der Wielen RP, Löwik MR, van den Berg $\mathrm{H}$, et al. Serum vitamin D concentrations among elderly people in Europe. Lancet. 1995; 346: 207-10.

18. Lips P, Duong T, Oleksik A, et al. A global study of vitamin D status and parathyroid function in postmenopausal women with osteoporosis: baseline data from the multiple outcomes of raloxifene evaluation clinical trial. J Clin Endocrinol Metab. 2001; 86: 121221.

19. Welsh P, Peters MJ, McInnes IB, et al. Vitamin $\mathrm{D}$ deficiency is common in patients with RA and linked to disease activity, but circulating levels are unaffected by TNFalpha blockade: results from a prospective cohort study. Ann Rheum Dis. 2011; 70: 1165-7.

20. Braun-Moscovici Y, Toledano K, Markovits $\mathrm{D}$, et al. Vitamin D level: is it related to disease activity in inflammatory joint disease? Rheumatol Int. 2011; 31: 493-9.

21. Haque UJ, Bartlett SJ. Relationships among vitamin $\mathrm{D}$, disease activity, pain and disability in rheumatoid arthritis. Clin Exp Rheumatol. 2010; 28: 745-7.

22. Kerr GS, Sabahi I, Richards JS, et al. Prevalence of vitamin D insufficiency/deficiency in rheumatoid arthritis and associations with disease severity and activity. J Rheumatol. 2011; 38: 53-9.

23. Kröger H, Penttilä IM, Alhava EM. Low serum vitamin D metabolites in women with rheumatoid arthritis. Scand J Rheumatol. 1993; 22: 172-7.

24. Reid D, Toole BJ, Knox S, et al. The relation between acute changes in the systemic inflammatory response and plasma 25-hydroxyvita- 
min D concentrations after elective knee arthroplasty. Am J Clin Nutr. 2011; 93: 1006-11.

25. Peelen E, Knippenberg S, Muris AH, et al. Effects of vitamin D on the peripheral adaptive immune system: a review. Autoimmun Rev. 2011; 10: 733-43.

26. Stockton KA, Mengersen K, Paratz JD, et al. Effect of vitamin D supplementation on muscle strength: a systematic review and metaanalysis. Osteoporos Int. 2011; 22: 859-71.

27. Wolfe F, Michaud K. Effect of body mass in- dex on mortality and clinical status in rheumatoid arthritis. Arthritis Care Res. 2012; 64: 1471-9.

28. Klaasen R, Wijbrandts CA, Gerlag DM, Tak PP. Body mass index and clinical response to infliximab in rheumatoid arthritis. Arthritis Rheum. 2011; 63: 359-64.

29. Adami S, Bertoldo F, Brandi ML, et al. Guidelines for the diagnosis, prevention and treatment of osteoporosis. Reumatismo. 2009; 61: 260-84. 